The Astrophysical Journal, 611:623-632, 2004 August 20

(C) 2004. The American Astronomical Society. All rights reserved. Printed in U.S.A.

\title{
LOW-FREQUENCY GRAVITATIONAL RADIATION FROM COALESCING MASSIVE BLACK HOLE BINARIES IN HIERARCHICAL COSMOLOGIES
}

\author{
Alberto Sesana, ${ }^{1}$ Francesco Haardt, ${ }^{1}$ Piero Madau, ${ }^{2}$ and Marta Volonteri ${ }^{2}$ \\ Received 2004 January 26; accepted 2004 April 23
}

\begin{abstract}
We compute the expected low-frequency gravitational wave signal from coalescing massive black hole (MBH) binaries at the center of galaxies in a hierarchical structure formation scenario in which seed holes of intermediate mass form far up in the dark halo "merger tree." The merger history of dark matter halos and associated MBHs is followed via cosmological Monte Carlo realizations of the merger hierarchy from redshift $z=20$ to the present in a $\Lambda \mathrm{CDM}$ cosmology. MBHs get incorporated through halo mergers into larger and larger structures, sink to the center because of dynamical friction against the dark matter background, accrete cold material in the merger remnant, and form MBH binary systems. Stellar dynamical (three-body) interactions cause the hardening of the binary at large separations, while gravitational wave emission takes over at small radii and leads to the final coalescence of the pair. A simple scheme is applied in which the "loss cone" is constantly refilled and a constant stellar density core forms because of the ejection of stars by the shrinking binary. The integrated emission from inspiraling MBH binaries at all redshifts is computed in the quadrupole approximation and results in a gravitational wave background (GWB) with a well-defined shape that reflects the different mechanisms driving the late orbital evolution. The characteristic strain spectrum has the standard $h_{c}(f) \propto f^{-2 / 3}$ behavior only in the range $f=10^{-9}$ to $10^{-6} \mathrm{~Hz}$. At lower frequencies the orbital decay of MBH binaries is driven by the ejection of background stars ("gravitational slingshot"), and the strain amplitude increases with frequency, $h_{c}(f) \propto f$. In this range the GWB is dominated by $10^{9}-10^{10} M_{\odot} \mathrm{MBH}$ pairs coalescing at $0 \lesssim z \lesssim 2$. At higher frequencies, $f>10^{-6} \mathrm{~Hz}$, the strain amplitude, as steep as $h_{c}(f) \propto f^{-1.3}$, is shaped by the convolution of last stable circular orbit emission by lighter binaries $\left(10^{2}-10^{7} M_{\odot}\right)$ populating galaxy halos at all redshifts. We discuss the observability of inspiraling MBH binaries by a low-frequency gravitational wave experiment such as the planned Laser Interferometer Space Antenna (LISA). Over a 3 yr observing period LISA should resolve this GWB into discrete sources, detecting $\approx 60(\approx 250)$ individual events above an $\mathrm{S} / \mathrm{N}=5(\mathrm{~S} / \mathrm{N}=1)$ confidence level.
\end{abstract}

Subject headings: black hole physics — cosmology: theory — early universe — gravitational waves relativity

\section{INTRODUCTION}

Studies of gravitational wave (GW) emission and its detectability are becoming increasingly topical in astrophysics. Technological developments of bars and interferometers bring the promise of a future direct observation of gravitational radiation, allowing us to test one of the most fascinating predictions of general relativity and, at the same time, providing a new powerful tool in the astronomical investigation of highly relativistic catastrophic events, such as the merging of compact binary systems and the collapse of massive stellar cores.

Massive black hole (MBH) binaries are among the primary candidate sources of GWs at $\mathrm{mHz}$ frequencies (see, e.g., Haehnelt 1994; Jaffe \& Backer 2003; Wyithe \& Loeb 2003), the range probed by the space-based Laser Interferometer Space Antenna (LISA). Today, MBHs are ubiquitous in the nuclei of nearby galaxies (see, e.g., Magorrian et al. 1998). If MBHs were also common in the past (as implied by the notion that many distant galaxies harbor active nuclei for a short period of their life), and if their host galaxies experience multiple mergers during their lifetime, as dictated by popular cold dark matter (CDM) hierarchical cosmologies, then MBH

\footnotetext{
${ }^{1}$ Dipartimento di Fisica e Matematica, Universitá dell'Insubria, via Valleggio 11, 22100 Como, Italy.

2 Department of Astronomy and Astrophysics, University of California, 1156 High Street, Santa Cruz, CA 95064.
}

binaries will inevitably form in large numbers during cosmic history. $\mathrm{MBH}$ pairs that are able to coalesce in less than a Hubble time will give origin to the loudest GW events in the universe.

Provided wide MBH binaries do not "stall," their GWdriven inspiral will follow the merger of galaxies and pregalactic structures at high redshifts. A low-frequency detector like LISA will be sensitive to waves from coalescing binaries with total masses in the range $10^{3}-10^{6} M_{\odot}$ out to $z \sim 5-10$ (Hughes 2002). Two obvious outstanding questions are then how far up in the dark halo merger hierarchy MBHs form, and whether stellar dynamical processes can efficiently drive wide MBH binaries to the GW emission stage. The luminous $z \approx 6$ quasars discovered in the Sloan Digital Sky Survey (Fan et al. 2001) imply that black holes more massive than a few billion solar masses were already assembled when the universe was less than a billion years old. These MBHs could arise naturally from the growth by Eddington-limited gas accretion of seed stellar-mass or intermediate-mass holes forming at $z \gtrsim 10$ (Haiman \& Loeb 2001). It seems also clear that, following the merger of two halo+MBH systems of comparable mass ("major mergers"), dynamical friction will drag in the "satellite" halo (and its MBH) toward the center of the more massive progenitor: this will lead to the formation of a bound MBH binary in the violently relaxed core of the newly merged stellar system. What remains uncertain is the demography of the MBH population as a function of redshift 
and the dynamics of MBH binaries, i.e., whether binaries will "hang up" before the back-reaction from GW emission becomes important.

In this paper we study the expected gravitational wave signal from inspiraling binaries in a hierarchical structure formation scenario in which seed holes of intermediate mass form far up in the dark halo "merger tree." The model has been discussed in detail in Volonteri et al. (2003a, hereafter Paper I). Seed holes are placed within rare high-density regions (minihalos) above the cosmological Jeans and cooling masses at redshift 20. Their evolution and growth are followed through Monte Carlo realizations of the halo merger hierarchy combined with semianalytical descriptions of the main dynamical processes, such as dynamical friction against the dark matter background, the shrinking of $\mathrm{MBH}$ binaries via threebody interactions, their coalescence due to the emission of gravitational waves, and the "gravitational rocket." Major mergers lead to $\mathrm{MBH}$ fueling. The long dynamical frictional timescales leave many MBHs "wandering" in galaxy halos after a minor merger. Bound pairs form after major mergers and lose orbital angular momentum by capturing stars passing within a distance of the order of the binary semimajor axis and ejecting them at much higher velocities. The heating of the surrounding stars by a decaying $\mathrm{MBH}$ pair creates a lowdensity core out of a preexisting stellar cusp, slowing down further binary hardening (see, e.g., Milosavljevic \& Merritt 2001). The model reproduces rather well the observed luminosity function of optically selected quasars in the redshift range $1<z<5$ (Paper I) and provides a quantitative explanation of the stellar cores observed today in bright elliptical galaxies as a result of the cumulative eroding action of shrinking MBH binaries (Volonteri et al. 2003b, hereafter Paper II).

The paper is organized as follows. In $\S 2$ we describe our merger tree algorithm coupled with semianalytical recipes designed to track the dynamical history of MBHs in a cosmological framework. We compute black hole binary coalescence rates as a function of redshift and mass and show that MBH binaries will shrink to the gravitational wave emission stage by scattering off background stars from stellar cusps. In $\S 3$ we summarize the basic theory of GW emission. In $\S 4$ we compute the cosmological GW background (GWB) from inspiraling $\mathrm{MBH}$ binaries and discuss the observability of individual coalescence events by LISA. Finally, we summarize our main results in $\S 5$. Unless otherwise stated, all results shown below refer to the currently favored $\Lambda$ CDM world model with $\Omega_{M}=0.3, \quad \Omega_{\Lambda}=0.7, \quad h=0.7, \quad \Omega_{b}=0.045$, $\sigma_{8}=0.93$, and $n=1$.

\section{MBH BINARIES}

\subsection{Assembly and Growth of MBHs}

Following Papers I and II, we track backward the merger history of parent halos with present-day masses in the range $10^{11}-10^{15} M_{\odot}$ with a Monte Carlo algorithm based on the extended Press-Schechter formalism (see, e.g., Cole et al. 2000). The tree is averaged over a large number of different realizations for a total of 220 simulated halos, and the results are weighted by the Press-Schechter halo mass function at the chosen output redshift. Pregalactic seed holes form with intermediate masses $\left(m_{\mathrm{BH}}=150 M_{\odot}\right)$ as remnants of the first generation of massive metal-free stars with $m_{*}>260 M_{\odot}$ that do not disappear as pair-instability supernovae (Madau \& Rees 2001). We place them in isolation within halos above
$M_{\text {seed }}=1.6 \times 10^{7} M_{\odot}$ collapsing at $z=20$ (the highest redshift that computational costs allow us to follow the merger hierarchy to) from rare greater than $3.5 \sigma$ peaks of the primordial density field. While $Z=0$ stars with $40 M_{\odot}<$ $m_{*}<140 M_{\odot}$ are also predicted to collapse to MBHs with masses exceeding half of the initial stellar mass (Heger \& Woosley 2002), we find that the GWB signal in the LISA window is not very sensitive to the precise choice for the seed hole mass.

Hydrodynamic simulations of major mergers have shown that a significant fraction of the gas in interacting galaxies falls to the center of the merged system (Mihos \& Hernquist 1996): the cold gas may be eventually driven into the very inner regions, fueling an accretion episode and the growth of the nuclear MBH (see, e.g., Kauffmann \& Haehnelt 2000). Since the local MBH mass density is consistent with the integrated luminosity density of quasars (Yu \& Tremaine 2002), the fraction of cold gas ending up in the hole must depend on the properties of the host halo in such a way as to ultimately lead to the observed correlation between stellar velocity dispersion and SMBH mass. As in Papers I and II, we assume that in each major merger (defined here as a merger between halos of mass ratio greater than 0.3 ), the more massive hole accretes, at the Eddington rate, a gas mass that scales with the fifth power of the circular velocity of the host halo,

$$
\Delta m_{\mathrm{acc}}=3.6 \times 10^{6} M_{\odot} \mathcal{K} V_{c, 150}^{5.2}
$$

where $V_{c, 150}$ is the circular velocity of the merged system in units of $150 \mathrm{~km} \mathrm{~s}^{-1}$. This relation follows from combining the $m_{\mathrm{BH}}-\sigma_{*}$ and the $V_{c}-\sigma_{*}$ relations given in Ferrarese (2002; see Papers I and II). The normalization factor $\mathcal{K}$ is of order unity and is fixed a posteriori in order to reproduce the correlation between stellar velocity dispersion and nuclear black hole mass observed today in nearby galaxies (Ferrarese \& Merritt 2000; Gebhardt et al. 2000).

Inside a halo of mass $M$, the dark matter is distributed according to an NFW profile (Navarro et al. 1997),

$$
\rho_{\mathrm{DM}}(r)=\frac{M}{4 \pi r\left(r+r_{\mathrm{vir}} / c\right)^{2} f(c)},
$$

where $r_{\text {vir }}$ is the virial radius, $c$ is the halo concentration parameter, and $f(c)=\ln (1+c)-c /(1+c)$. The distribution of concentrations and its scaling with halo mass and redshift of collapse are taken from Bullock et al. (2001). Dynamical friction drags satellite progenitors - and their MBHs if they host one or more-toward the center of the more massive preexisting system on a timescale that depends on the orbital parameters of the satellites (van den Bosch et al. 1999) and on tidal mass loss/evaporation (Taffoni et al. 2003). The initial occupation fraction of seed holes is so small that the merging of two halos both hosting a black hole is a rare event even with the assumed "bias," and MBHs evolve largely in isolation. At redshifts below 15 , however, more than $10 \%$ of hosts contain two or more MBHs, and bound binaries begin forming in significant numbers.

The evolution of a bound binary is determined by the initial central stellar distribution. We model this as a (truncated) singular isothermal sphere with one-dimensional velocity dispersion $\sigma_{*}$ and density

$$
\rho_{*}(r)=\frac{\sigma_{*}^{2}}{2 \pi G r^{2}} .
$$


The stellar velocity dispersion is related to the halo circular velocity $V_{c}$ at the virial radius following Ferrarese (2002),

$$
\log V_{c}=(0.88 \pm 0.17) \log \sigma_{*}+(0.47 \pm 0.35) .
$$

\subsection{Binary Merger Rates}

The semimajor axis $a(t)$ of a bound binary continues to shrink because of dynamical friction from distant stars acting on each MBH individually, until it becomes "hard" when the separation falls below (Quinlan 1996)

$$
a_{h}=\frac{G m_{2}}{4 \sigma_{*}^{2}}=0.2 \mathrm{pc} m_{6} \sigma_{70}^{-2}
$$

where $m_{2}\left(<m_{1}\right)$ is the mass of the lighter hole, $m_{6} \equiv$ $m_{2} / 10^{6} M_{\odot}$, and $\sigma_{70}$ is the stellar velocity dispersion in units of $70 \mathrm{~km} \mathrm{~s}^{-1}$. After this stage the MBH pair hardens via threebody interactions, i.e., by capturing and ejecting at much higher velocities the stars passing by within a distance $\sim a$ (Begelman et al. 1980). We assume that the "bottleneck" stages of binary shrinking occur for separations $a<a_{h}$; during a galactic merger, after a dynamical friction timescale, we place the MBH pair at $a_{h}$ and let it evolve. The hardening of the binary modifies the stellar density profile, removing mass interior to the binary orbit, depleting the galaxy core of stars, and slowing down further hardening. If $\mathcal{M}_{\mathrm{ej}}$ is the stellar mass ejected by the pair, the binary evolution and its effect on the galaxy core are determined by two dimensionless quantities: the hardening rate

$$
H=\frac{\sigma_{*}}{G \rho_{*}} \frac{d}{d t} \frac{1}{a}
$$

and the mass ejection rate

$$
J=\frac{1}{\left(m_{1}+m_{2}\right)} \frac{d \mathcal{M}_{\mathrm{ej}}}{d \ln (1 / a)} .
$$

The quantities $H$ and $J$ can be found from scattering experiments that treat the test particle-binary encounters one at a time (Quinlan 1996). We assume as in Papers I and II (see also Merritt 2000) that the stellar mass removal creates a core of radius $r_{c}$ and constant density $\rho_{c} \equiv \rho_{*}\left(r_{c}\right)$, so that the total mass ejected as the binary shrinks from $a_{h}$ to $a$ can be written as

$$
\mathcal{M}_{\mathrm{ej}}=\frac{2 \sigma_{*} 2}{G}\left(r_{c}-r_{i}\right)+M_{i}-M_{c}=\frac{4}{3} \frac{\sigma_{*}^{2}\left(r_{c}-r_{i}\right)}{G}
$$

where $M_{c}=4 \pi \rho_{c} r_{c}^{3} / 3, M_{i}=4 \pi \rho_{i} r_{i}^{3} / 3, r_{i}=r_{c}(t=0)$ is the radius of the (preexisting) core when the hardening phase starts at $t=0$, and $\rho_{i} \equiv \rho_{*}\left(r_{i}\right)$. The core radius then grows as

$$
r_{c}(t)=r_{i}+\frac{3}{4 \sigma_{*}^{2}} G\left(m_{1}+m_{2}\right) \int_{a(t)}^{a_{h}} \frac{J(a)}{a} d a .
$$

The binary separation quickly falls below $r_{c}$, and subsequent evolution is slowed down because of the declining stellar density, with a hardening time

$$
t_{h}=\left|\frac{a}{\dot{a}}\right|=\frac{2 \pi r_{c}(t)^{2}}{H \sigma_{*} a}
$$

that becomes increasingly long as the binary shrinks. The mass ejected increases approximately logarithmically with time, and the binary "heats" background stars at radii $r_{c} \gg a$.
We assume that the effect of the hierarchy of MBH binary interactions is cumulative (the "core preservation" model of Paper II); i.e., the mass displaced by the pair is not replenished after every major merger event, and $r_{i}$ continues to grow during the cosmic evolution of the host. This is supported by $N$-body simulations involving mergers of spherical galaxy models with different density profiles and showing that the remnant profile is quite close to the profile of the progenitors - in other words, that the core appears to be preserved during such mergers (e.g., Fulton \& Barnes 2001). The above scheme can be modified by rare triple black hole interactions, when another major merger takes place before the preexisting binary has had time to coalesce (see, e.g., Hut $\&$ Rees 1992). In this case there is a net energy exchange between the binary and the third incoming black hole, resulting in the ejection of the lighter hole and the recoil of the binary. The binary also becomes more tightly bound. The reader is referred to Paper I for a detailed discussion of the role played by triple interactions. If hardening continues down to a separation,

$$
a_{\mathrm{gw}}=0.0014 \mathrm{pc}\left[\frac{\left(m_{1}+m_{2}\right) m_{1} m_{2}}{10^{18.3} M_{\odot}^{3}}\right]^{1 / 4} t_{9}^{1 / 4},
$$

the binary will coalesce within $t_{9}$ Gyr because of the emission of gravitational waves. An equal-mass pair must then manage to shrink by a factor

$$
\frac{a_{h}}{a_{\mathrm{gw}}} \approx 150 m_{6}^{3 / 4} \sigma_{70}^{-2} t_{9}^{-1 / 4}
$$

for gravity wave emission to become efficient. Since the hardening and mass ejection rate coefficients are $H \approx 15$ and $J \approx 1$ in the limit of a very hard binary (Quinlan 1996), the hardening time in equation (10) can be rewritten (for $m_{1}=m_{2}$ and $r_{i} \rightarrow 0$ ) as

$$
\begin{aligned}
t_{h} & \approx 4.7 \times 10^{4} \mathrm{yr} m_{6} \sigma_{70}^{-3}\left(a_{h} / a\right) \ln ^{2}\left(a_{h} / a\right) \\
& \approx 5.6 \times 10^{4} \mathrm{yr} m_{6}^{0.35}\left(a_{h} / a\right) \ln ^{2}\left(a_{h} / a\right),
\end{aligned}
$$

where the second equality assumes the $m_{\mathrm{BH}}-\sigma_{*}$ relation of Ferrarese (2002). Allowing for the cumulative effect of a hierarchy of $\mathrm{MBH}$ binary interactions (i.e., for the existence of a preexisting core of radius $r_{i}$ ) increases somewhat the hardening time in equation (13). Yet, we find $t_{h}$ to be comparable to or shorter than the then Hubble time at all but the highest redshifts. Figure 1 shows the number of $\mathrm{MBH}$ binary coalescences per unit redshift per unit observed year, $d N / d z d t$, predicted by our model. The observed event rate is obtained by dividing the rate per unit proper time by the $(1+z)$ cosmological time dilation factor. Each panel shows the rate for different $m_{\mathrm{BH}}=m_{1}+m_{2}$ mass intervals and lists the integrated event rate, $d N / d t$, across the entire sky. The number of events per observed year per unit redshift peaks at $z=2$ for $10^{7} M_{\odot}<m_{\mathrm{BH}}<10^{9} M_{\odot}$, at $z=3-4$ for $10^{5} M_{\odot}<m_{\mathrm{BH}}<$ $10^{7} M_{\odot}$, and at $z=10$ for $m_{\mathrm{BH}}<10^{5} M_{\odot}$; i.e., the lower the black hole mass, the higher the peak redshift. Beyond the peak, the event rate decreases steeply with cosmic time. Previous calculations of this quantity have either assumed instantaneous black hole coalescence and computed halo merger rates only out to $z \sim 5$ (Menou et al. 2001), assigned a constant (independent of black hole mass, background stellar density, and redshift) efficiency factor to the coalescence process (Wyithe \& Loeb 2003), or used phenomenological 


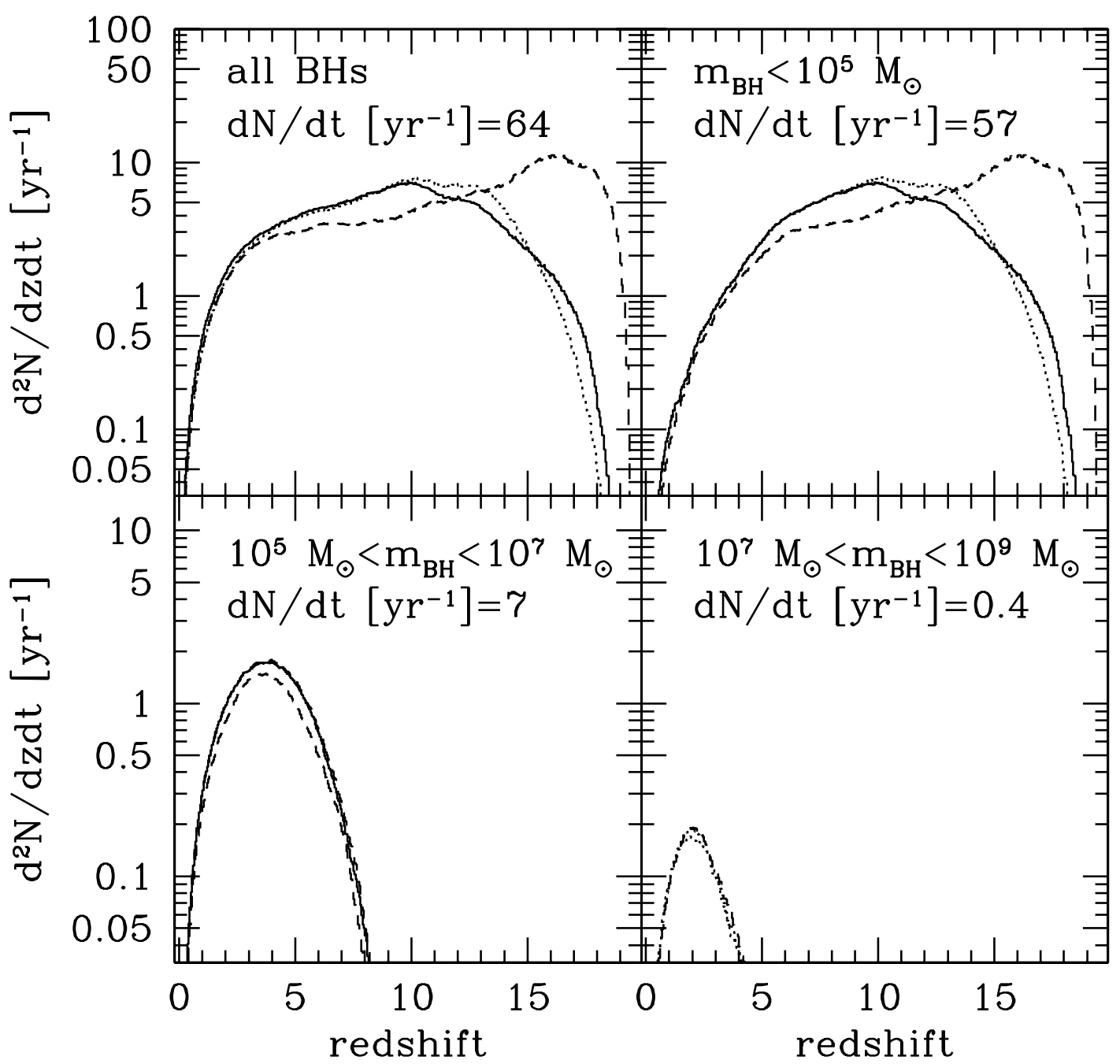

FIG. 1.-Number of MBH binary coalescences observed per year at $z=0$, per unit redshift, in different $m_{\mathrm{BH}}=m_{1}+m_{2}$ mass intervals. Each panel also lists the integrated event rate, $d N / d t$, predicted by our model. The rates (solid lines) are compared to a case in which triple black hole interactions are switched off (dotted lines). Triple black hole interactions increase the coalescence rate at very high redshifts, while for $10<z<15$, the rate is decreased because of the reduced number of surviving binaries. Dashed lines: Rates computed assuming binary hardening is instantaneous, i.e., MBHs coalesce after a dynamical friction timescale.

and empirical rates (Jaffe \& Backer 2003). Our rates are significantly smaller than those computed by Wyithe \& Loeb (2003), consistent with their assumption that all MBH binaries actually coalesce.

While our calculations are the first to include a detailed model of MBH binaries dynamics, we note that our scheme implicitly neglects the depopulation of the "loss cone" (Frank \& Rees 1976), since it is the total stellar density that is allowed to decrease following equation (9), not the density of low angular momentum stars. The effect of loss-cone depletion (the depletion of low angular momentum stars that get close enough to extract energy from a hard binary) has traditionally been one of the major uncertainties in constructing viable merger scenarios for MBH binaries (Begelman et al. 1980). Several processes have recently been studied that should all mitigate the problems associated with loss-cone depletion, such as the wandering of the binary center of mass from the galaxy center induced by continuous interactions with background stars (Chatterjee et al. 2003), the large supply of low angular momentum stars in significantly flattened or triaxial galaxies ( $\mathrm{Yu}$ 2002), the presence of a third hole (Blaes et al. 2002), and the randomization of stellar orbits due to the infall of small satellites (Zhao et al. 2002).
Our scheme also neglects the role of gaseous - rather than stellar dynamical-processes in driving the evolution of an MBH binary (see, e.g., Escala et al. 2004). We will try to address the effect of some these uncertainties on the predicted GWB in $\S 4$.

There is another process that our hierarchical MBH evolution scenario includes: this is the "gravitational rocket," the recoil due to the nonzero net linear momentum carried away by GWs in the coalescence of two unequal mass black holes. Radiation recoil is a strong field effect that depends on the lack of symmetry in the system and may displace MBHs from galaxy centers or even eject them into intergalactic space (Redmount \& Rees 1989). Its outcome is still uncertain, as fully general relativistic numerical computations of radiation reaction effects are not available at the moment. Here we have used the extrapolated perturbative results on test mass particles of Fitchett \& Detweiler (1984), predicting in a Schwarzschild geometry recoil velocities larger than $200 \mathrm{~km} \mathrm{~s}^{-1}$ only for mass ratios $m_{2} / m_{1}>0.4$. As shown in Figure 2, in our model the typical mass ratio of merging binaries exceeds 0.4 at $z>10$. It is at early times then that the gravitational rocket is effective at ejecting MBHs from the shallow potential wells (escape velocities 

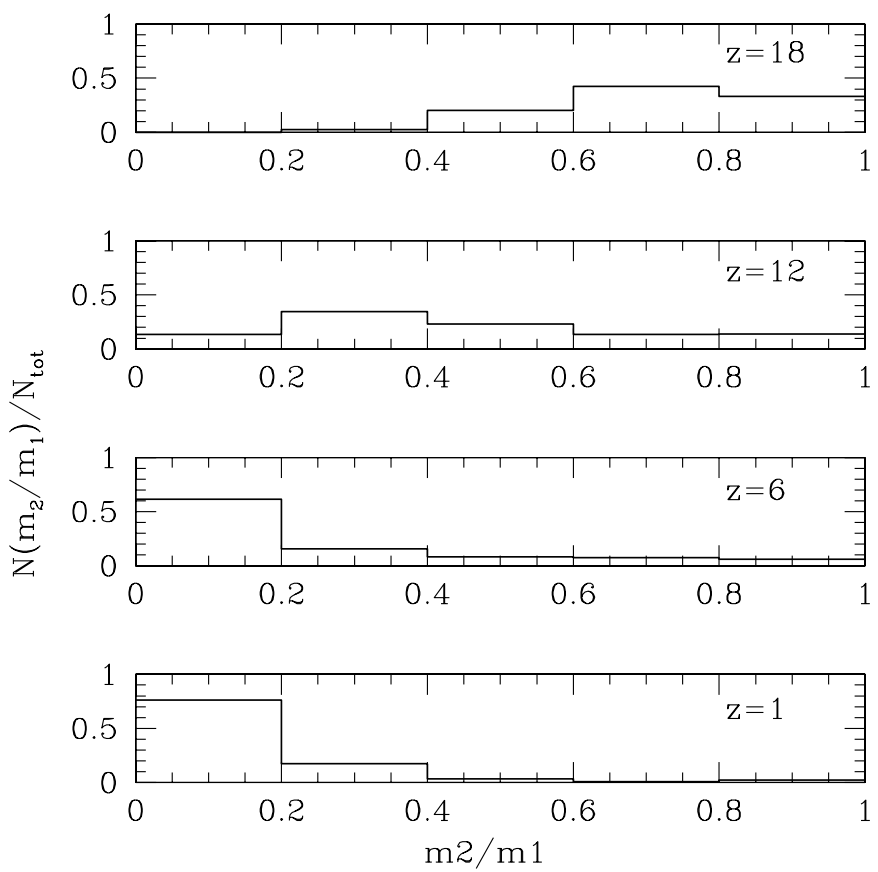

FIG. 2.- Normalized distribution of mass ratios of coalescing MBH binaries at different epochs, averaged over 220 realizations. At very high redshift, binary members are seed holes with nearly equal masses; as time goes on, MBHs grow as a result of gas accretion, and low mass ratios become more probable.

less than $100 \mathrm{~km} \mathrm{~s}^{-1}$ ) of their hosts (Madau et al. 2004). Overall, we find that $\approx 25 \%$ of coalesced pairs recoil and escape their host halos at $z \sim 10$ and that the ejected holes have typical masses of $\gtrsim 1000 M_{\odot}$. The detectability of recoiling MBHs has recently been addressed by Madau \& Quataert (2004).

\section{GRAVITATIONAL WAVE SIGNAL}

\subsection{Basic Theory}

The theory of GW emission in the quadrupole approximation is a textbook topic (e.g., Schutz \& Ricci 2001; Maggiore 2000 ), and we recall here only the basic relations. The Einstein equations can be linearized over the perturbed Minkowski metric. The perturbed part of the metric or "strain" admits a wave solution out of the source of the stress-energy tensor. Such solution becomes particularly easy to treat in the socalled transverse-traceless (TT) gauge. In the TT gauge the strain at distance $r$ from the source has the form

$$
\begin{gathered}
h_{\mu 0}^{\mathrm{TT}}=0, \\
h_{i k}^{\mathrm{TT}}(t, r)=\frac{2 G}{c^{4} r}\left[\frac{d^{2}}{d t^{2}} Q_{i k}^{\mathrm{TT}}\left(t-\frac{r}{c}\right)\right],
\end{gathered}
$$

so that only the spatial components are not vanishing. The term $Q_{i k}^{\mathrm{TT}}$ is the reduced quadrupole momentum projected into the TT gauge, containing the stress-energy tensor of the gravitational source and evaluated at the retarded time $t-r / c$.

It is then possible to associate an energy momentum pseudotensor to the wave, which, to first perturbative order, transforms as a tensor. Such a pseudotensor is

$$
t_{\mu \nu}=\frac{c^{4}}{32 \pi G}\left\langle h_{i j, \mu}^{\mathrm{TT}} h_{, \nu}^{\mathrm{TT} i j}\right\rangle,
$$

where \langle\rangle is an average over wavelength. If we consider a GW propagating along the positive $x$-direction, then the associated energy flux will be

$$
c t_{01}=\frac{d E_{\mathrm{gw}}}{d t d S}=\frac{c^{5}}{32 \pi G}\left\langle h_{i j, 0}^{\mathrm{TT}} h_{, 1}^{\mathrm{TT} i j}\right\rangle .
$$

Taking equation (14), deprojecting the quadrupole momentum $Q^{\mathrm{TT}}$ out of the TT gauge to get $Q$, and integrating over the surface $S$, one derives the luminosity associated with GW emission,

$$
\frac{d E_{\mathrm{gw}}}{d t}=\int_{S} \frac{d E_{\mathrm{gw}}}{d t d S} d S=\frac{G}{5 c^{5}}\left\langle\frac{d^{3}}{d t^{3}} Q_{i j} \frac{d^{3}}{d t^{3}} Q^{i j}\right\rangle .
$$

This depends on the time third derivative of the (reduced) quadrupole momentum of the source.

\subsection{Gravitational Waves from Binary Systems}

In the stationary case, i.e., assuming no orbital decay, the GW emission spectrum of an $\mathrm{MBH}$ pair in a circular orbit of radius $a$ is a delta function at rest-frame frequency $f_{r}=$ $\omega / \pi$, where $\omega=\left[G\left(m_{1}+m_{2}\right) / a^{3}\right]^{1 / 2}$ is the Keplerian angular frequency of the binary. Orbital decay (due to GW emission and/or the ejection of background stars) results in a shift of the emitted frequency to increasingly larger values as the binary evolution proceeds. The energy spectrum integrated over the entire radiating lifetime of the source (a continuum formed by the superposition of delta functions) can be computed once the energy emitted per logarithmic frequency interval,

$$
\frac{d E_{\mathrm{gw}}}{d \ln f_{r}}=\frac{d E_{\mathrm{gw}}}{d t} \frac{d t}{d a} \frac{d a}{d f_{r}} f_{r},
$$

is known. This is related to the characteristic strain, $h_{c}$, through

$$
\frac{d E_{\mathrm{gw}}}{d \ln f_{r}} \propto f_{r}^{2} h_{c}^{2}\left(f_{r}\right)
$$

where $h_{c}$ is defined as

$$
\left\langle h_{\alpha \beta}(t) h^{\alpha \beta}(t)\right\rangle=2 \int\left(d \ln f_{r}\right) h_{c}^{2}\left(f_{r}\right) .
$$

The characteristic strain is related to the Fourier transform of the strain $\tilde{h}\left(f_{r}\right), h_{c}^{2}\left(f_{r}\right)=2 f_{r}\left|\tilde{h}\left(f_{r}\right)\right|^{2}$. In the case of a binary system equation (17) can be rewritten as

$$
\frac{d E_{\mathrm{gw}}}{d t}=\frac{32 \pi^{10 / 3} G^{7 / 3}}{5 c^{5}}\left(\mathcal{M} f_{r}\right)^{10 / 3} .
$$

The radiated power is an increasing function of frequency and of the "chirp mass" of the system,

$$
\mathcal{M} \equiv \frac{m_{1}^{3 / 5} m_{2}^{3 / 5}}{\left(m_{1}+m_{2}\right)^{1 / 5}}
$$

The term $d a / d f$ in equation (18) is readily obtained by differentiating the relation $f_{r}=\omega / \pi$,

$$
\frac{d a}{d f_{r}}=-\frac{2}{3}\left[\frac{G\left(m_{1}+m_{2}\right)}{\pi^{2}}\right]^{1 / 3} f_{r}^{-5 / 3} .
$$


Finally, the term $d a / d t$ represents the orbital decay due to angular momentum losses. When the back-reaction from GW emission dominates, the orbit shrinks at a rate

$$
\left(\frac{d a}{d t}\right)_{\mathrm{gw}}=-\frac{64}{5} \frac{G^{3}}{c^{5} a^{3}} m_{1} m_{2}\left(m_{1}+m_{2}\right),
$$

and the resulting spectrum of GWs is

$$
\left(\frac{d E_{\mathrm{gw}}}{d \ln f_{r}}\right)_{\mathrm{gw}}=\frac{(\pi G)^{2 / 3}}{3} \mathcal{M}^{5 / 3} f_{r}^{2 / 3} .
$$

In the early stages of binary evolution, however, angular momentum losses are driven by stellar dynamical processes, and the emerging GW spectrum will show a different scaling with frequency. The model outlined in $\S 2.1$ leads to the following expression for the orbital decay rate in the "gravitational slingshot" regime (eq. [10]):

$$
\left(\frac{d a}{d t}\right)_{\mathrm{gs}}=-\frac{H \sigma_{*} a^{2}}{2 \pi r_{c}^{2}} .
$$

Assuming an initially cuspy profile $\left(r_{i}=0\right)$ and in the limit of a very hard binary $(J \approx 1)$, the core radius (eq. [9]) created by the shrinking pair can be written as

$$
r_{c}(t) \approx \frac{3}{4 \sigma_{*}^{2}} G\left(m_{1}+m_{2}\right) \ln \left(\frac{a_{h}}{a}\right),
$$

and the hardening rate becomes proportional (neglecting the logarithmic dependence) to

$$
\left(\frac{d a}{d t}\right)_{\mathrm{gs}} \propto \sigma_{*}^{5}\left(m_{1}+m_{2}\right)^{-2} a^{2} .
$$

Contrary to the case in which GW losses dominates, the binary decay slows down with time because of the declining stellar density, and the rate $|d a / d t|$ decreases as the orbit shrinks. Inserting the above expression into equation (18) yields the following scaling for the emitted GW spectrum in the stellar ejection regime:

$$
\left(\frac{d E_{\mathrm{gw}}}{d \ln f_{r}}\right)_{\mathrm{gs}} \propto m_{1}^{2} m_{2}^{2}\left(m_{1}+m_{2}\right) \sigma_{*}^{-5} f_{r}^{4} .
$$

The GW spectrum arising from a binary system during the slow inspiral phase spans a broad but finite interval of emitted frequencies, $f_{\min }<f_{r}<f_{\max }$. We assume that the binary begins emitting GWs at separation $a=a_{h}$, so that the lower limit $f_{\min }$ is

$$
f_{\min }=\frac{8 \sigma_{*}^{3}}{\pi G}\left(m_{1}+m_{2}\right)^{1 / 2} m_{2}^{-3 / 2} .
$$

The upper limit $f_{\max }$ is set by the frequency emitted at $a=6 G m_{1} / c^{2}$, the radius of the closest stable circular orbit for two nonrotating holes,

$$
f_{\max }=\frac{c^{3}}{6^{3 / 2} \pi G}\left(m_{1}+m_{2}\right)^{1 / 2} m_{1}^{-3 / 2} .
$$

We do not address in this work the higher frequency radiation emitted during the subsequent coalescence and ringdown phases (Flanagan \& Hughes 1998). Figure 3 shows three examples of GW spectra emitted by inspiraling binaries. The

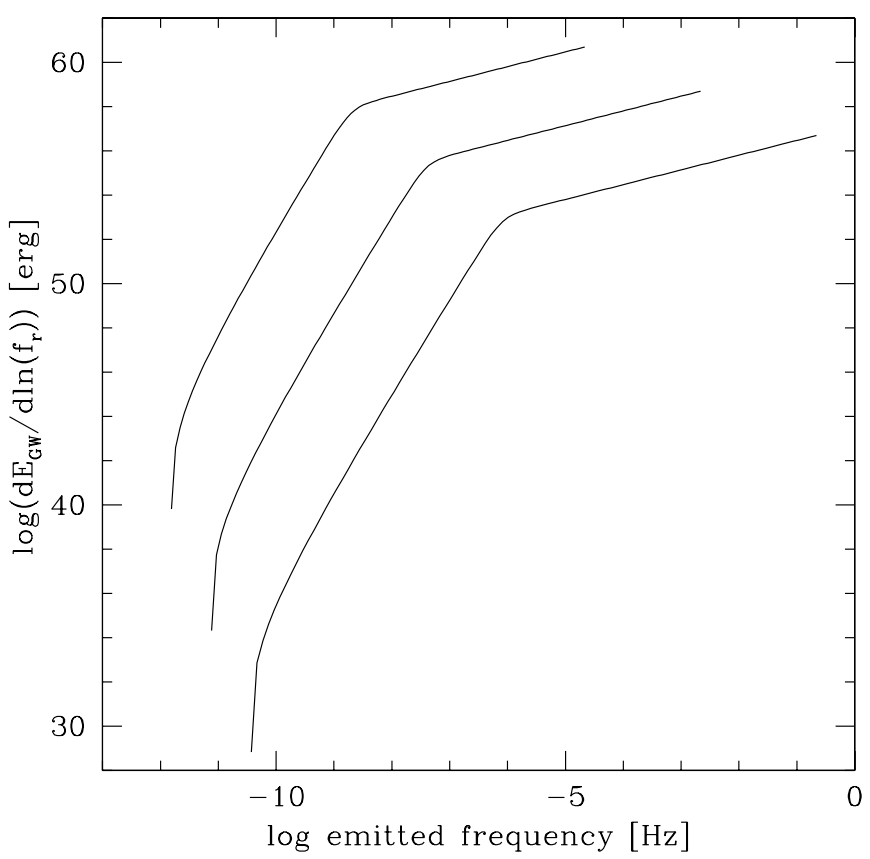

FIG. 3.-Energy per natural log frequency interval around $f_{r}$ emitted in GWs by an equal-mass $\left(m_{1}=m_{2}\right)$ black hole binary system in a stellar background of velocity dispersion $\sigma_{*}$. From top to bottom, the three curves correspond to $\left(m_{1}, \sigma_{*}\right)=\left(10^{8} M_{\odot}, 180 \mathrm{~km} \mathrm{~s}^{-1}\right),\left(10^{6} M_{\odot}, 66 \mathrm{~km} \mathrm{~s}^{-1}\right)$, and $\left(10^{4} M_{\odot}, 24 \mathrm{~km} \mathrm{~s}^{-1}\right)$, respectively.

two regimes, stellar slingshot at low frequencies and GW losses at high frequencies, are clearly recognizable.

\subsection{Total Background from Cosmic Sources}

We can now compute the total background-random GWs arising from a large number of independent events-produced by a population of sources at cosmological distances. Following Phinney (2001), the present-day energy density in GWs of frequency $f$ per natural log frequency interval is

$$
c^{2} \rho_{\mathrm{gw}}(f)=\frac{\pi}{4} \frac{c^{2}}{G} f^{2} h_{c}^{2}(f)=\int_{0}^{\infty} d z \frac{N(z)}{1+z} \frac{d E_{\mathrm{gw}}}{d \ln f_{r}},
$$

where $N(z) d z$ is the comoving number density of catastrophic events in the redshift interval $z, z+d z$, the factor $1 /(1+z)$ accounts for the redshifting of gravitons, and the observed frequency $f$ is related to the rest-frame frequency $f_{r}$ by $f=f_{r} /(1+z)$. Equation (32) assumes that all sources emit the same intrinsic spectrum (i.e., that all binaries have the same mass and shrink in the same stellar background) and that the inspiraling timescale is much shorter than the then Hubble time (i.e., that the inspiral occurs at the same redshift as the coalescence). When binary decay occurs on timescales that are comparable or longer than the expansion time, equation (32) can be generalized as

$$
c^{2} \rho_{\mathrm{gw}}(f)=f \int_{0}^{\infty} d z N\left(f_{r}, t\right) \frac{d E_{\mathrm{gw}}}{d t}\left|\frac{d t}{d z}\right|,
$$

where $N\left(f_{r}, t\right)$ is the comoving number density of binaries at cosmic time $t$ that radiate at frequencies between $f_{r}$ and $f_{r}+d f_{r}, d t / d z=-(1+z)^{-1} H(z)^{-1}$, where $H(z)$ is the Hubble parameter and $d E_{\mathrm{gw}} / d t$ is the GW luminosity at $f_{r}=f(1+z)$ measured in the rest frame of the pair. The density of sources $N\left(f_{r}\right)$ is related to the space density of binaries with separations between $a$ and $a+d a$ by $N\left(f_{r}\right) d f_{r}=-N(a) d a$. If $\dot{N}_{b}$ is 
the birth rate (after dynamical friction) of $\mathrm{MBH}$ binaries and $\dot{N}_{c}$ is the rate of coalescences, then $N(a)$ is given by the continuity equation

$$
\frac{\partial N}{\partial t}+\frac{\partial(\dot{a} N)}{\partial a}=\dot{N}_{b} \delta\left(a-a_{h}\right)-\dot{N}_{c} \delta(a)
$$

Note that since in the situation we are considering the sources of GWs are $\mathrm{MBH}$ binaries with a mass function that changes with cosmic epoch, the term $N\left(f_{r}, t\right) d E_{\mathrm{gw}} / d t$ must be summed up over all binary types.

\subsection{Resolvable Signal}

While the data stream of LISA will include confusion noise arising from a large number of unresolved Galactic and extragalactic white dwarf binaries (Farmer \& Phinney 2003; Nelemans et al. 2001), the GWB from MBH binaries will be resolvable into discrete sources at least in the high-frequency end of the LISA sensitivity range. The nature (stochastic or resolved) of the GWB can be assessed by counting the number of events per resolution frequency bin whose observed signal is larger than a given sensitivity threshold $h_{c, \text { min }}$. The background is resolved if such number is less than unity and the cumulative signal from all sources below threshold is negligible.

The resolution frequency bin is set by the minimum frequency resolvable by a given mission, and it is simply the inverse of the mission lifetime. The characteristic strain $h_{c}$ of a source at comoving coordinate distance $r(z)$ is

$$
h_{c} \simeq h \sqrt{n}
$$

where the strain amplitude $h$ (sky- and polarization-averaged) is given by

$$
h=\frac{8 \pi^{2 / 3}}{10^{1 / 2}} \frac{G^{5 / 3} \mathcal{M}^{5 / 3}}{c^{4} r(z)} f_{r}^{2 / 3}
$$

and $n$ is the number of cycles a source spends at frequency $f_{r}$, i.e., $n=f_{r}^{2} / \dot{f}_{r}$ (Thorne 1987). ${ }^{3}$ Note that for a finite observation time $\tau$, the number of cycles actually observed at frequency $f_{r}$ cannot exceed $f \tau$. The frequency evolution $\dot{f}_{r}$ can be obtained by combining equations (23) and (24) in the GW regime and equations (23) and (26) in the slingshot regime. At the endpoint of the inspiraling phase, the number of cycles spent near that frequency is close to unity, so that $h_{c} \simeq h$.

\section{RESULTS}

\subsection{Gravitational Wave Background}

Merger tree realizations allow us to follow the time evolution of individual inspiraling binaries and to solve equation (33) by performing a direct weighted sum of the emission from individual evolving pairs. The resulting GWB is shown in Figure 4, where the square of the characteristic strain is plotted against observed frequency. The peak occurs for $f_{\text {peak }} \approx 10^{-10} \mathrm{~Hz}$. For $f \lesssim f_{\text {peak }}$ the spectrum is shaped by the evolution of binaries in the stellar slingshot regime, and according to equation (29), $h_{c}^{2} \propto f^{2}$ just below $f_{\text {peak }}$. At even lower frequencies the energy distribution is somewhat steeper

\footnotetext{
3 The formula for $h$ in Thorne (1987) contains a multiplicative extra factor $(4 / 3)^{1 / 2}$ to account for the Earth's rotation.
}

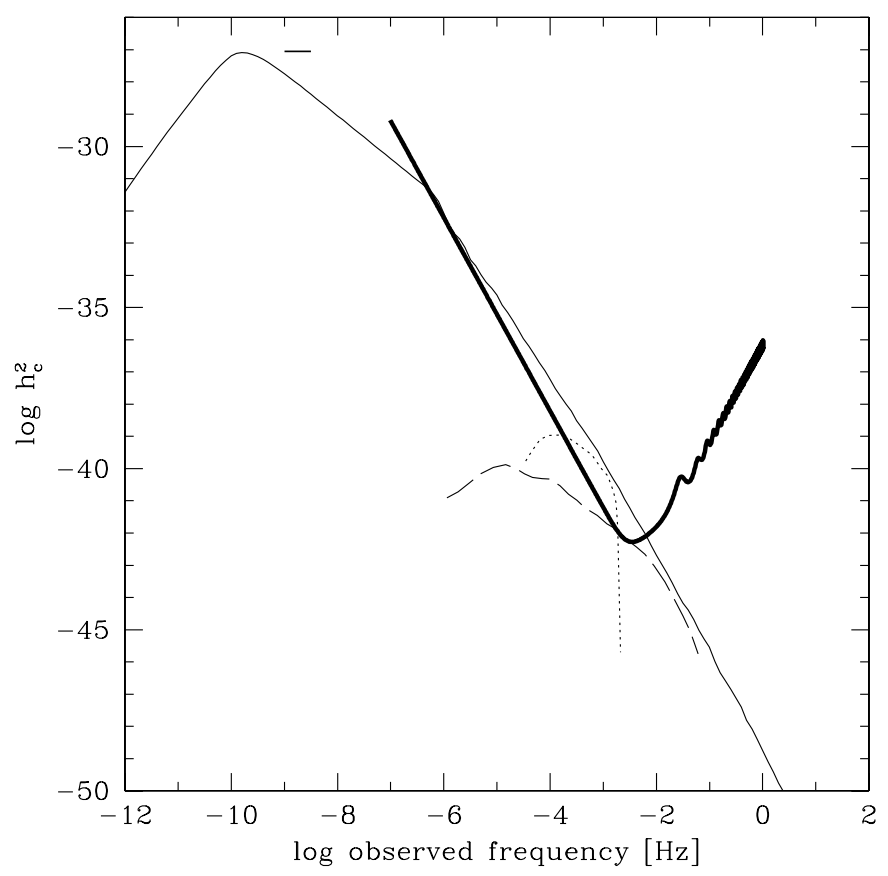

FIG. 4.-Gravitational wave background from inspiraling MBH binaries in the hierarchical scenario described in the text. The square of the characteristic strain (directly observable by a long-base interferometer) is plotted vs. wave frequency (solid line). Thick solid line: LISA single-arm Michelson sensitivity curve. Thick dash at $f \simeq 10^{-9}$ : Current limits from pulsar timing experiments. Long-dashed line at $10^{-6} \mathrm{~Hz} \lesssim f \lesssim 0.1 \mathrm{~Hz}$ : Expected strain from extragalactic white dwarf binaries (Farmer \& Phinney 2003). Dotted line at $10^{-4} \mathrm{~Hz} \lesssim$ $f \lesssim 10^{-3} \mathrm{~Hz}$ : Expected strain from unresolved Galactic white dwarf binaries (Nelemans et al. 2001).

than $f^{2}$ because of the assumed cutoff in the individual emission spectra for $a=a_{h}$ (see Fig. 3). For frequencies $f \gtrsim f_{\text {peak }}$ but below $10^{-6} \mathrm{~Hz}, h_{c}^{2} \propto f^{-4 / 3}$, as expected in the classical GW regime (see eqs. [19] and [25]). At even higher frequencies, $f \gtrsim 10^{-6} \mathrm{~Hz}$, the strain is shaped by the convolution of the sharp frequency cutoff at the last stable orbit of individual binaries (see Fig. 3), and in this range one has approximately $h_{c}^{2} \propto f^{-2.6}$. The slope in this regime is directly related to the rate of binary coalescences and to the $\mathrm{MBH}$ mass function.

Figure 4 also shows the LISA single-arm Michelson sensitivity curve for a sky- and polarization-averaged signal with a signal-to-noise ratio $(\mathrm{S} / \mathrm{N})=1{ }^{4}$ It is apparent how LISA could detect the GWB above $10^{-5} \mathrm{~Hz}$, while the peak at $\mathrm{nHz}$ frequencies is close to current limits of pulsar timing experiments (Lommen 2002). We have also plotted the GWB expected in the LISA window from the cosmological population of white dwarf binaries computed by Farmer \& Phinney (2003) and the GWB from unresolved Galactic white dwarf binaries (Nelemans et al. 2001). Our estimate of the background due to $\mathrm{MBH}$ binaries lies well above the expected strain from cosmological white dwarf binaries.

The contribution to the GWB from $\mathrm{MBH}$ binaries in different mass ranges is depicted in Figure 5, while Figure 6 shows the signal from different redshift intervals. From these figures it is clear how for $f \lesssim 10^{-6} \mathrm{~Hz}$ the background is dominated by events at moderate redshifts, $0 \lesssim z \lesssim 2$, from very massive binaries, $m_{1} \gtrsim 10^{8} M_{\odot}$.

\footnotetext{
${ }^{4}$ The curve is taken from http://www.srl.caltech.edu/ shane/sensitivity, where it is given in terms of the "effective strain" $h_{\text {eff }}=h_{c} / \sqrt{f}$.
} 


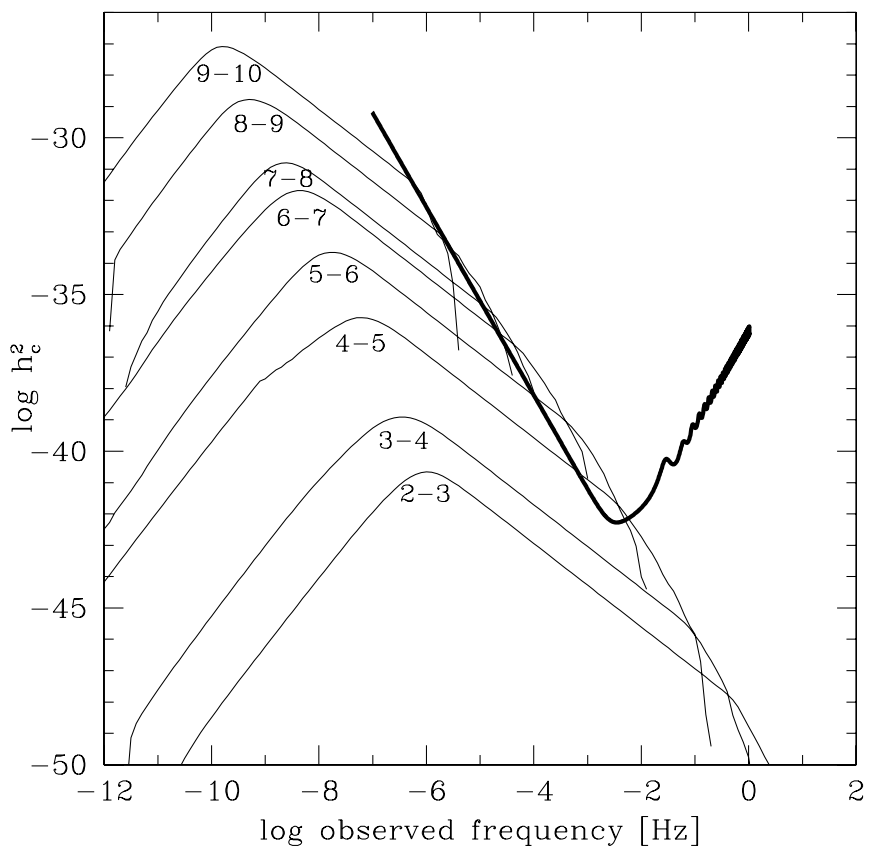

FIG. 5.- Integrated GWB from inspiraling MBH binaries in different mass ranges. From top to bottom (indicated by labels), the curves show the signal produced by events with $9<\log \left(m_{1} / M_{\odot}\right)<10,8<\log \left(m_{1} / M_{\odot}\right)<9, \ldots$, and $2<\log \left(m_{1} / M_{\odot}\right)<3$. Here $m_{1}$ is the mass of the heavier black hole.

Lighter binaries show up at higher frequencies, where the background drops in amplitude. For $f \gtrsim 10^{-4} \mathrm{~Hz}$, the signal is dominated by $m_{1} \lesssim 10^{7} M_{\odot}$ binaries, with comparable contributions from events at $z<3$ and $z>3$. The peak frequency $f_{\text {peak }}$ of each curve in Figure 6 is determined by two factors acting in opposite directions, i.e., the Hubble expansion and the time evolution of the binary mass function. The latter remains approximately constant in the redshift interval $0 \lesssim$ $z \lesssim 2$, so that the spectrum originating from sources at $1 \lesssim z \lesssim 2$ appears, because of the Hubble expansion, redshifted with respect to the signal from $0 \lesssim z \lesssim 1$ events. The peak frequency of events at $z \gtrsim 2$ becomes bluer because the effect of the reduced average binary mass at increasing redshifts dominates over the Hubble expansion.

\subsection{Stochastic versus Resolved Signal}

Merger tree realizations allow us to compute the GW signal from individual $\mathrm{MBH}$ binaries and to compare it with the LISA instrumental noise. Figure 7 depicts the number of systems above the $\mathrm{S} / \mathrm{N}=5$ and $\mathrm{S} / \mathrm{N}=1$ sensitivity thresholds, per resolution frequency bin $\left(\Delta f=10^{-8} \mathrm{~Hz}\right.$ assuming a $3 \mathrm{yr}$ mission duration) and shows that such number is less than unity all over the surveyed frequency range. For reference, we also plot the total number of sources per bin, i.e., the number of sources regardless of the strength of their GW signal. Even in this ideal case, for $f \gtrsim 10^{-3.5} \mathrm{~Hz}$, the GWB would be resolved into discrete events. We have also checked that the signal from the rare resolved events accounts for more than $99 \%$ of the total GWB, i.e., the numerous sources below the sensitivity threshold provide a negligible background at Earth. We conclude that the GWB from cosmological MBH binaries will be resolved into discrete events all over the LISA frequency range.

Integrating over frequencies, the number of $\mathrm{MBH}$ binary systems observable by LISA with $\mathrm{S} / \mathrm{N}$ in excess of $5(1)$ is $\approx 20$

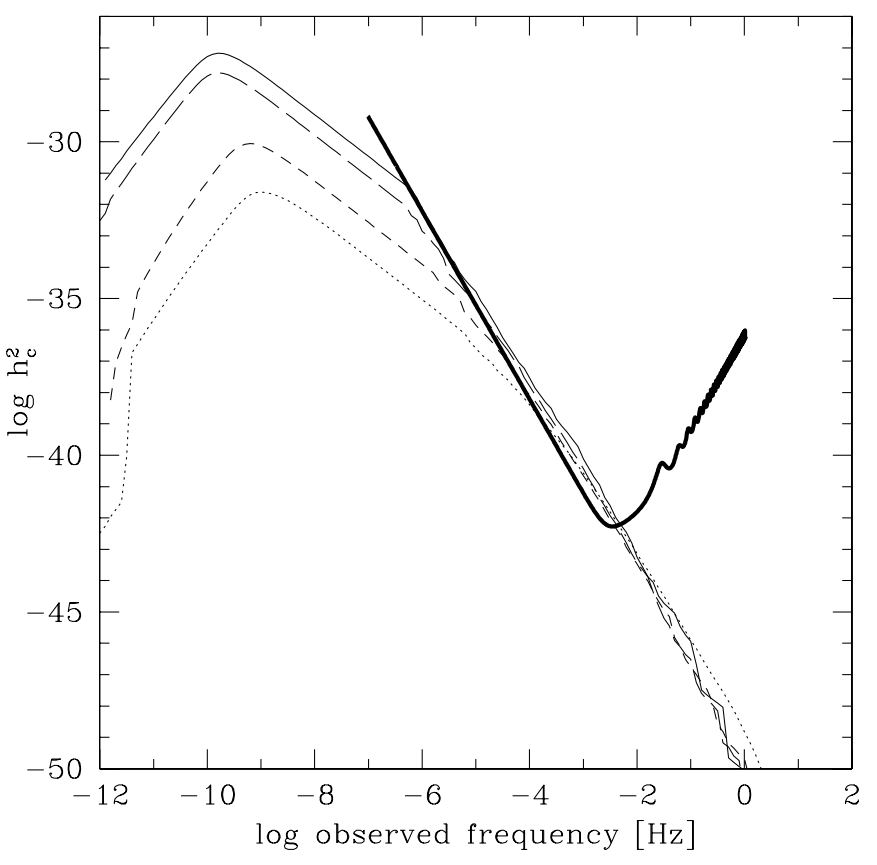

FIG. 6.-GWB from different redshift intervals. From top to bottom, the spectrum of the characteristic strain is computed integrating the emission from events at $0<z<1$ (solid line), $1<z<2$ (long-dashed line), $2<z<3$ (short-dashed line), and $z>3$ (dotted line), respectively.

(200). ${ }^{5}$ These numbers refer to stationary events, i.e., binaries at large separation whose GW signal does not shift much in frequency and hence is long-lasting compared to the

${ }^{5}$ The figures given are obtained neglecting every source of noise other than instrumental. In particular, the stochastic noise from Galactic WD-WD binaries shown in Fig. 4 has not been considered here.

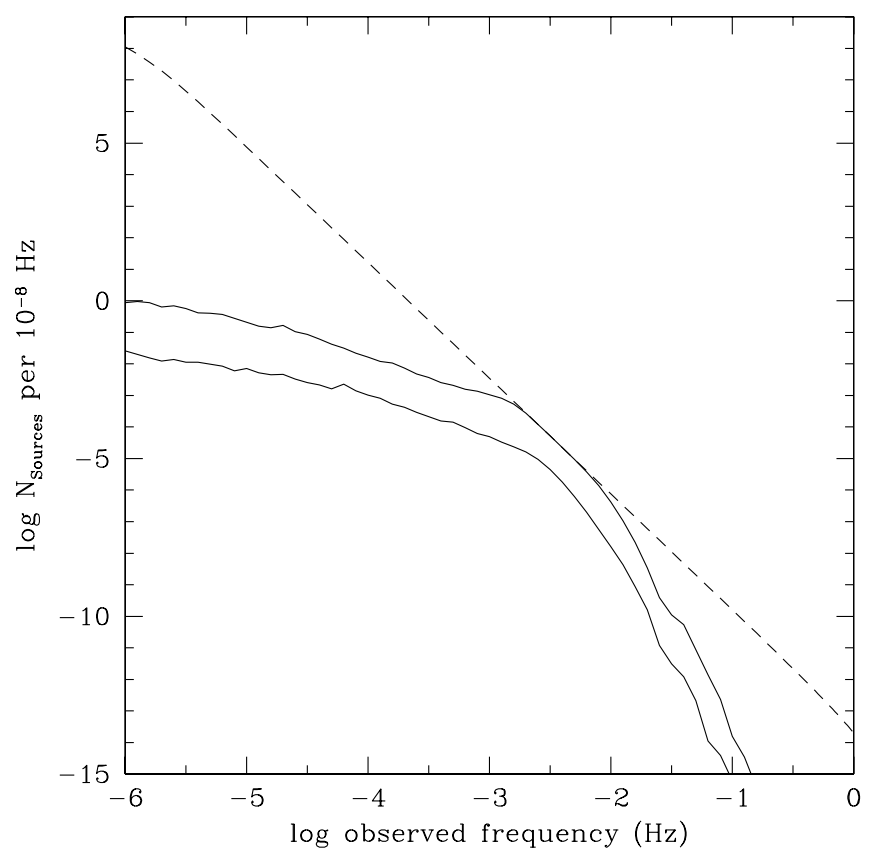

FIG. 7.-Number of $\mathrm{MBH}$ binaries per $10^{-8} \mathrm{~Hz}$ frequency bin contributing to the GWB. Dashed line: All binaries. Top solid line: Binaries above LISA $\mathrm{S} / \mathrm{N}=1$ sensitivity threshold. Bottom solid line: Binaries above LISA $\mathrm{S} / \mathrm{N}=5$ sensitivity threshold. 


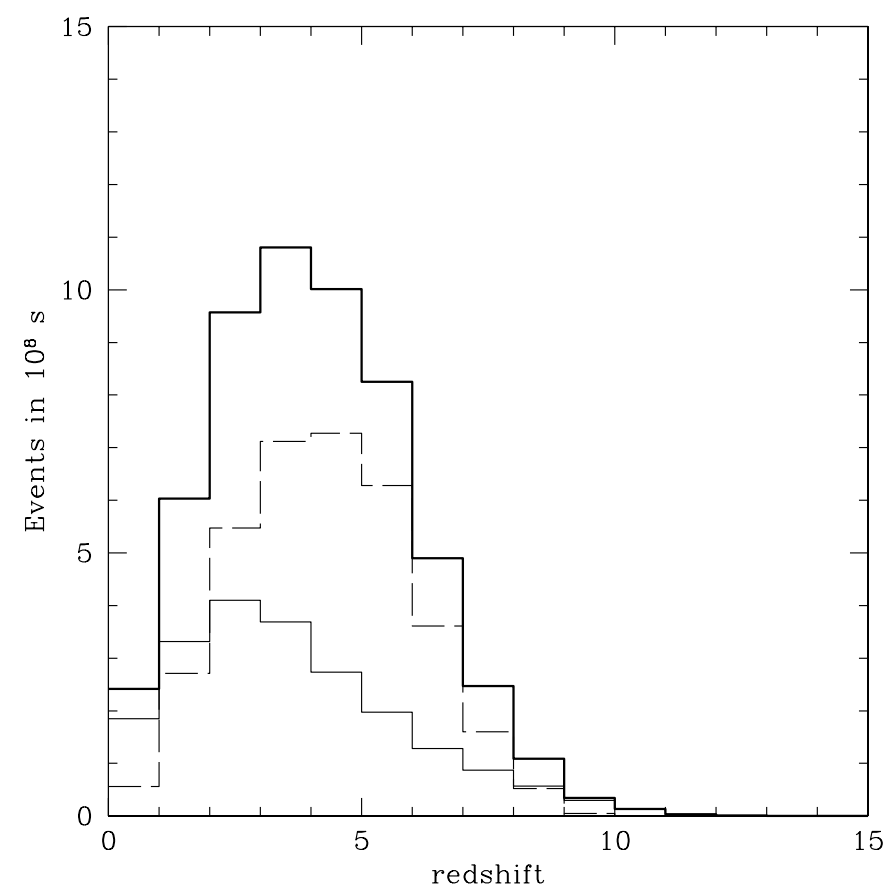

FIG. 8.-Number of events per unit redshift interval resolved by LISA with $\mathrm{S} / \mathrm{N}>5$ in $10^{8} \mathrm{~s}$. Thick solid histogram: Total number of events in $10^{8} \mathrm{~s}$. Solid histogram: Number of stationary events. These events are of much longer duration compared to the mission lifetime. Dashed histogram: Number of bursts in $10^{8} \mathrm{~s}$. These events are of short duration compared to the mission lifetime.

observation time. The number of such events is independent of any (plausible) mission duration. On the contrary, the number of observable bursts, i.e., binaries during the observation period, clearly depends on the mission lifetime. In $10^{8} \mathrm{~s}$ there will be $\approx 200$ bursts (see Fig. 1, top left panel), and among them, $\approx 35(50)$ are detectable by LISA with $\mathrm{S} / \mathrm{N}>5$ $(\mathrm{S} / \mathrm{N}>1)$.

The redshift distribution of detectable events is shown in Figure 8 , in the case of the $\mathrm{S} / \mathrm{N}=5$ threshold. The distribution of bursts is similar to that of coalescences shown in Figure 1 (bottom left panel), indicating that a relevant fraction of bursts at high redshifts produces a signal above threshold. The peak of the redshift distribution of stationary events is shifted at lower redshift by two different instrumental effects. First, the inclusion of a detection threshold selects preferentially binaries at low redshifts. Second, as for wide binaries the time spent at a given frequency can be larger than the observation time, only a fraction of the signal (see eqs. [35] and [36]) may actually be detected in a $10^{8} \mathrm{~s}$ observation. This fact causes the drop of the stationary event counts below $z \simeq 3$ seen in Figure 8.

\subsection{Test and Comparison}

It is fair at this stage to ask how sensitive our predictions are to the details and uncertainties of the adopted scheme for binary formation and evolution. To answer this question we have run two test models, arbitrarily dividing and multiplying the computed hardening time $t_{h}$ by a factor of 3 , i.e., significantly increasing or reducing the rate of binary decay by stellar dynamical processes.

In the "fast" hardening case, $f_{\text {peak }}$ increases by a factor $\sim 30 \%$ and the peak amplitude drops by $\sim 10 \%$. This is due to the fact that when $t_{h}$ is shorter, GW emission losses dominates over stellar slingshots only at shorter binary separations. The opposite occurs in the case of "slow" hardening, in which $f_{\text {peak }}$ is lowered by a factor $\sim 30 \%$ and the peak amplitude is $\sim 20 \%$ larger than in the standard case. For $f \gtrsim f_{\text {peak }}$, the background amplitude is $15 \%$ larger in the "fast" hardening case, because a larger number of $\mathrm{MBH}$ binaries can now coalesce. The coalescence rate is now 78 per observed year, compared to 64. Again, the opposite is true in the case of "slow" hardening, in which the coalescence rate decreases to 50 per observed year. The situation is different in the LISA window. A faster or slower hardening has basically no effects, as the increased or reduced rate of coalescence affects very massive binaries only, which do not contribute to the LISA frequency range. Consequently, the precise value of the hardening time has no significant impact on the number of sources (either stationary or bursts) observable by LISA. This is, of course, only true as long as $t_{h}$ is less than the then Hubble time. If MBH binaries were instead to "stall" (because of the depopulation of the loss cone), the rate of detectable events could become negligible. We also find our predictions to be sensitive to the occupation fraction of halos hosting nuclear MBHs. Within our assumptions (rare "high $\sigma$ " seed holes), this is of order $50 \%$ at $z \sim 5$ for halos more massive than $10^{10} M_{\odot}$. A lower occupation fraction would decrease the rate of inspiraling events, at the expense of making MBHs nonubiquitous in the nuclei of nearby galaxies. The black hole coalescing rate would be higher if the seeds were more common at early times.

We have compared our results with the calculations of Wyithe \& Loeb (2003), who estimated the GWB from MBH binaries using a different, but similar in spirit, approach. In their work the time-dependent binary mass function is explicitly derived from the extended Press-Schechter formalism. The nature of such methodology hampers the possibility of following the evolution of individual $\mathrm{MBH}$ pairs (e.g., triple interactions as well as the differential frequency broadening due to the nonnegligible duration of the orbital decay phase are neglected). The two estimates of the GWB appear in good agreement (to within a factor of 2) at high frequencies ( $f \gtrsim 10^{-4} \mathrm{~Hz}$ ), a fact reflecting the common hierarchical scenario, the similar normalization to the present-day $m_{\mathrm{BH}}-\sigma_{*}$ relation, and our relatively short binary decay timescales. By contrast, our predicted number counts are about an order of magnitude smaller than those given by Wyithe \& Loeb (2003). This is because they do not consider the shift in frequency during the inspiraling phase and compute the counts at a fixed frequency of $10^{-3} \mathrm{~Hz}$. Their simplified treatment has the effect of artificially boosting (by as much as a factor of $\sim 50$ ) the number of detectable events at $z \gtrsim 7$.

Finally, we were concerned that in the LISA window the GWB we computed is entirely due to GWs emitted at the last stable orbit of the coalescing pair, a regime the Newtonian approximation may fail to describe adequately. To quantify this we have implemented a post-Newtonian (of order 2) approximation describing the GW emission from binary systems (Blanchet 2001). We find that differences in the spectrum of a coalescing $\mathrm{MBH}$ binary are quite small, of order $10 \%$, and the overall results are basically unaffected.

\section{SUMMARY AND CONCLUSIONS}

We have computed the gravitational wave signal (in terms of the characteristic strain spectrum) from the cosmological 
population of inspiraling $\mathrm{MBH}$ binaries predicted to form at the center of galaxies in a hierarchical structure formation scenario. The assembly and growth of MBHs was followed using Monte Carlo realizations of the halo merger hierarchy from the present epoch to very high redshifts, coupled with semianalytical recipes treating the dynamics of the (inevitably forming) $\mathrm{MBH}$ binaries. We find that the broadband GWB spectrum can be divided into three different regimes. For frequencies $\lesssim 10^{-10} \mathrm{~Hz}$, the GWB is shaped by $\mathrm{MBH}$ binaries in the gravitational slingshot regime, i.e., the orbital decay is driven by scattering off background stars. In the intermediate band, $10^{-9} \mathrm{~Hz} \lesssim f \lesssim 10^{-6} \mathrm{~Hz}$, GW emission itself dominates potential energy losses and the strain has the "standard" $f^{-2 / 3}$ behavior. Finally, for $f \gtrsim 10^{-6}$, the background signal is formed by the convolution of the emission at the last stable orbit from individual binaries. In the first two regimes, the strain is dominated by low-redshift events $(z \lesssim 2)$ involving genuinely supermassive holes. At larger frequencies the main contribution comes from lighter and lighter binaries. Such background should dominate that predicted from a population of extragalactic white dwarf binaries. As already pointed out by Wyithe \& Loeb (2003), in the $\mathrm{nHz}$ regime probed by pulsar timing experiments, the amplitude of the characteristic strain from coalescing $\mathrm{MBH}$ binaries is close to current experimental limits (Lommen 2002).

In the LISA window $\left(10^{-6} \mathrm{~Hz} \lesssim f \lesssim 0.1 \mathrm{~Hz}\right)$, the main sources of GWs are MBH binaries in the mass range $10^{3} M_{\odot} \lesssim$ $m_{1} \lesssim 10^{7} M_{\odot}$. With a plausible lifetime of $\simeq 3 \mathrm{yr}, L I S A$ will resolve the GWB into $\approx 60$ discrete sources above the $\mathrm{S} / \mathrm{N}=5$ confidence level. Among these, $\approx 35$ are bursts, i.e., binaries caught in their final inspiraling phase. We predict that most of the observable events will be at redshifts $2 \lesssim z \lesssim 7$, with only a handful at larger redshifts. While LISA will make it possible to probe the coalescence of early black hole binaries in the universe, it may not be able to observe the formation epochs of the first MBHs.

We have benefited from several discussions with V. Gorini. Support for this work was provided by NASA grant NAG511513 and NSF grant AST 02-05738 (P. M.).
Begelman, M. C., Blandford, R. D., \& Rees, M. J. 1980, Nature, 287, 307 Blaes, O., Lee, M. H., \& Socrates, A. 2002, ApJ, 578, 775

Blanchet, L. 2001, in Gravitational Waves, ed I. Ciufolini, V. Gorini, U. Moschella, \& P. Fré (Bristol: IOP), 338

Bullock, J. S., Kolatt, T. S., Sigad, Y., Somerville, R. S., Kravtsov, A. V.,

Klypin, A. A., Primack, J. R., \& Dekel, A. 2001, MNRAS, 321, 559

Chatterjee, P., Hernquist, L., \& Loeb, A. 2003, ApJ, 592, 32

Cole, L., Lacey, C. G., Baugh, C. M., \& Frenk, C. S. 2000, MNRAS, 319, 168 Escala, A., Larson, R. B., Coppi, P. S., \& Mardones, D. 2004, ApJ, 607, 765 Fan, X., et al. 2001, AJ, 122, 2833

Farmer, A. J., \& Phinney, E. S. 2003, MNRAS, 346, 1197

Ferrarese, L. 2002, ApJ, 578, 90

Ferrarese, L., \& Merritt, D. 2000, ApJ, 539, L9

Fitchett, M., \& Detweiler, S. 1984, MNRAS, 211, 933

Flanagan, E. E., \& Hughes, S. A. 1998, Phys. Rev. D, 57, 4535

Frank, J., \& Rees, M. J. 1976, MNRAS, 176, 633

Fulton, E., \& Barnes, J. E. 2001, Ap\&SS, 276, 851

Gebhardt, K., et al. 2000, ApJ, 543, L5

Haehnelt, M. G. 1994, MNRAS, 269, 199

Haiman, Z., \& Loeb, A. 2001, ApJ, 552, 459

Heger, A., \& Woosley, S. E. 2002, ApJ, 567, 532

Hughes, S. A. 2002, MNRAS, 331, 805

Hut, P., \& Rees, M. J. 1992, MNRAS, 259, 27P

Jaffe, A. H., \& Backer, D. C. 2003, ApJ, 583, 616

Kauffmann, G., \& Haehnelt, M. G. 2000, MNRAS, 311, 576

Lommen, A. N. 2002, in Proc. 270 WE-Heraeus Seminar on Neutron Stars, Pulsars, and Supernova Remnants, ed. W. Becker, H. Lesch, \& J. Trümper (MPE Rep. 278; Garching: MPE), 114

Madau, P., \& Quataert, E. 2004, ApJ, 606, L17
REFERENCES

Madau, P., \& Rees, M. J. 2001, ApJ, 551, L27

Madau, P., Rees, M. J., Volonteri, M., Haardt, F., \& Oh, S. P. 2004, ApJ, 604, 484

Maggiore, M. 2000, Phys. Rep., 331, 283

Magorrian, J., et al. 1998, AJ, 115, 2285

Menou, K., Haiman, Z., \& Narayanan, V. K. 2001, ApJ, 558, 535

Merritt, D. 2000, in ASP Conf. Ser. 197, Dynamics of Galaxies: From the Early Universe to the Present, ed. F. Combes, G. A. Mamon, \& V. Charmandaris (San Francisco: ASP), 221

Mihos, J. C., \& Hernquist, L. 1996, ApJ, 464, 641

Milosavljevic, M., \& Merritt, D. 2001, ApJ, 563, 34

Navarro, J. F., Frenk, C. S., \& White, S. D. M. 1997, ApJ, 490, 493

Nelemans, G., Yungelson, L. R., \& Portegies Zwart, S. F. 2001, A\&A, 375, 890

Phinney, E. S. 2001, MNRAS, submitted (astro-ph/0108028)

Quinlan, G. D. 1996, NewA, 1, 35

Redmount, I. H., \& Rees, M. J. 1989, Comments Astrophys., 14, 165

Schutz, B. F., \& Ricci, F. 2001, in Gravitational Waves, ed I. Ciufolini, V. Gorini, U. Moschella, \& P. Fré (Bristol: IOP), 11

Taffoni, G., Mayer, L., Colpi, M., \& Governato, F. 2003, MNRAS, 341, 434

Thorne, K. S. 1987, in 300 Years of Gravitation, ed. S. Hawking \& W. Israel (Cambridge: Cambridge Univ. Press), 330

van den Bosch, F. C., Lewis, G. F., Lake, G., \& Stadel, J. 1999, ApJ, 515, 50

Volonteri, M., Haardt, F., \& Madau, P. 2003a, ApJ, 582, 559 (Paper I)

Volonteri, M., Madau, P., \& Haardt, F. 2003b, ApJ, 593, 661 (Paper II)

Wyithe, J. S. B., \& Loeb, A. 2003, ApJ, 590, 691

Yu, Q. 2002, MNRAS, 331, 935

Yu, Q., \& Tremaine, S. 2002, MNRAS, 335, 965

Zhao, H. S., Haehnelt, M. G., \& Rees, M. J. 2002, NewA, 7, 385 